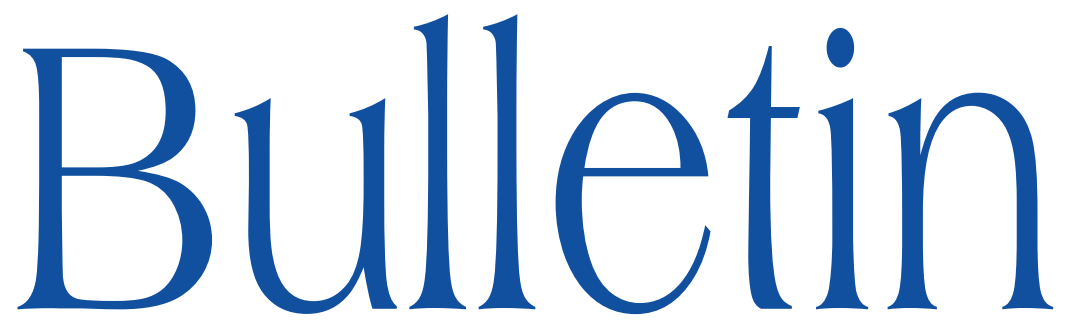

de la SOCIÉTÉ MATHÉMATIQUE DE FRANCE

\title{
COMPARAISON ENTRE COHOMOLOGIE CRISTALLINE ET COHOMOLOGIE ÉTALE $p$-ADIQUE SUR CERTAINES VARIÉTÉS DE SHIMURA
}

Tome 137

Sandra Rozensztajn

Fascicule 3

2000 


\title{
COMPARAISON ENTRE COHOMOLOGIE CRISTALLINE ET COHOMOLOGIE ÉTALE $p$-ADIQUE SUR CERTAINES VARIÉTÉS DE SHIMURA
}

\author{
PAR SANDRA ROZENSZTAJN
}

\begin{abstract}
Résumé. - Soit $X$ un modèle entier en un premier $p$ d'une variété de Shimura de type PEL, ayant bonne réduction associée à un groupe réductif $G$. On peut associer aux $\mathbb{Z}_{p}$-représentations du groupe $G$ deux types de faisceaux : des cristaux sur la fibre spéciale de $X$, et des systèmes locaux pour la topologie étale sur la fibre générique. Nous établissons un théorème de comparaison entre la cohomologie de ces deux types de faisceaux.
\end{abstract}

Abstract (Comparison between crystalline cohomology and p-adic étale cohomology on certain Shimura varieties)

Let $X$ be an integral model at a prime $p$ of a Shimura variety of PEL type having good reduction, associated to a reductive group $G$. To $\mathbb{Z}_{p}$ representations of the group $G$ can be associated two kinds of sheaves: crystals on the special fiber of $X$, and locally constant étale sheaves on the generic fiber. We establish a comparison between the cohomology of these two kinds of sheaves.

\section{Introduction}

L'objet de cet article est de montrer comment des théorèmes de comparaison « classiques » (c'est-à-dire pour la cohomologie à valeur dans des faisceaux

Texte reçu le 27 avril 2007, révisé le 19 décembre 2008, accepté le 2 mars 2009

Sandra Rozensztajn, Université de Lyon, UMPA, ENS Lyon, 46 allée d'Italie, 69007 Lyon, France - E-mail : rozenszt@umpa.ens-lyon.fr

Classification mathématique par sujets (2000). - 14G35,14F30,14F20.

Mots clefs. - Variétés de Shimura, Théorie de Hodge $p$-adique. 
constants) peuvent être utilisés pour montrer des théorèmes de comparaison pour certains faisceaux sur des variétés de Shimura.

Considérons $X$ un modèle entier d'une variété de Shimura de type PEL, défini sur une extension de $\mathbb{Z}_{p}$. Cette variété de Shimura correspond à un groupe réductif $G$, défini sur $\mathbb{Z}_{(p)}$. On peut associer aux $\mathbb{Z}_{(p)}$-représentations du groupe $G$ différents faisceaux : des systèmes locaux en $\mathbb{Z}_{p}$-modules sur la fibre générique de $X$, et des cristaux sur sa fibre spéciale.

La théorie de Hodge $p$-adique nous fournit des théorèmes de comparaison entre cohomologie étale $p$-adique et log-cristalline dans le cas des coefficients constants, pour des schémas propres et possédant certaines propriétés de lissité. Ces résultats sont dûs entre autres à Tsuji ([15]) pour le cas où l'on considère les groupes de cohomologie après tensorisation par $\mathbb{Q}_{p}$, et à Tsuji et Breuil pour le cas où l'on tient compte de la torsion ([16] et [2]). Dans ce dernier cas, il y a alors des restrictions essentielles sur le degré des groupes de cohomologie que l'on peut comparer. Ces théorèmes sont rappelés dans le paragraphe 5.1.

Nous utilisons ces théorèmes pour démontrer des théorèmes de comparaison entre la cohomologie étale du système local associé à une représentation $V \mathrm{du}$ groupe $G$, et la cohomologie log-cristalline d'une extension du cristal associé à $V$ à une compactification appropriée de $X$.

Le principe de notre méthode est de considérer la cohomologie à coefficients constants de la variété abélienne universelle sur $X$ et de ses puissances, et d'en déduire la comparaison qui nous intéresse en découpant les groupes de cohomologie des faisceaux considérés dans les groupes de cohomologie à coefficients constants à l'aide de certaines correspondances algébriques. Afin de mener à bien cette stratégie, nous avons besoin de plusieurs ingrédients :

1. l'existence de bonnes compactifications de $X$ et de certaines variétés de Kuga-Sato ;

2. des théorèmes de comparaison appropriés en théorie de Hodge $p$-adique;

3. une manière de travailler efficacement avec les faisceaux associés à la représentation $V$.

Concernant le premier point : les théorèmes de comparaison dont on a besoin n'étant valables que sur des schémas propres, nous devons supposer l'existence (prouvée dans certains cas seulement) de compactifications non seulement de $X$, mais aussi des variétés de Kuga-Sato, et plus précisément un système projectif de telles compactifications. La partie 1 explique précisément dans quelle situation nous nous plaçons, ainsi que les propriétés des compactifications que nous utilisons.

Concernant le deuxième point : la restriction (essentielle) sur le degré des groupes de cohomologie que l'on peut comparer impose des restrictions sur les

TOME $137-2009-\mathrm{N}^{\mathrm{O}} 3$ 
représentations $V$ auxquelles on peut appliquer notre méthode ainsi que sur le degré de la cohomologie que l'on considère.

Concernant le troisième point : nous devons nous restreindre aux représentations de $G$ qui donnent lieu à des faisceaux dont la cohomologie peut être découpée par des correspondances algébriques dans la cohomologie de la variété abélienne universelle. Ceci nous amène à définir dans la partie 2 la notion de représentation atteignable. Cette partie utilise de manière essentielle la structure des représentations du groupe réductif $G$, ce qui explique que l'on soit obligé de faire une description au cas par cas. Nous détaillons dans cette partie le cas des groupes unitaires et le cas Siegel.

Le plan de l'article est dès lors assez naturel. Dans la partie 1, nous donnons des rappels sur les variétés de Shimura de type PEL et nous expliquons quelles sont les compactifications dont on a besoin, et lesquelles sont connues. Dans la partie 2, nous définissons ce que sont les représentations atteignables de $G$. Nous expliquons ensuite dans la partie 3 l'action des correspondances algébriques ainsi que la construction des faisceaux associés aux représentations de $G$. Dans la partie 4, nous montrons que les groupes de cohomologie des faisceaux que l'on a construits sont munis des structures habituelles (frobenius, filtration, ...). Enfin, la partie 5 contient l'énoncé précis et la démonstration des théorèmes que l'on obtient (ce sont les théorèmes 5.3 et 5.4). C'est ici qu'apparaît une différence entre les cas unitaire et Siegel : en effet le point-clé de la preuve est la compatibilité de l'action des correspondances algébriques que nous considérons avec les théorèmes de comparaison à coefficients constants. Pour certaines représentations dans le cas Siegel, on utilise la compatibilité de cet isomorphisme de comparaison avec les structures produit sur les groupes de cohomologie, ce qui n'est démontré que dans le cas rationnel et non dans le cas de torsion. Dans le cas unitaire et pour les autres représentations dans le cas Siegel, on n'utilise pas la structure produit et on obtient un résultat qui tient compte de la torsion.

L'intérêt de cette comparaison est qu'il est possible d'obtenir des renseignements sur le côté cristallin : des techniques de type complexe BGG, décrites par exemple dans le chapitre VI de [4] permettent d'avoir des renseignements sur la filtration de Hodge. On en déduit alors des informations sur le côté étale, vu comme représentation galoisienne. 


\section{Les variétés considérées}

\subsection{Variétés de Shimura de type PEL}

\subsubsection{Les données}

On se donne $B$ une $\mathbb{Q}$-algèbre simple finie, munie d'une involution positive notée $*$ (c'est-à-dire que $\operatorname{tr}_{B / \mathbb{Q}}\left(x x^{*}\right)>0$ pour tout $x$ non nul de $\left.B\right), \mathfrak{V}$ un module de type fini sur $B$, muni d'une forme bilinéaire $($,$) telle que pour tous$ $v$ et $w$ dans $\mathfrak{V}$, et tout $b$ dans $B$ on ait $(b v, w)=\left(v, b^{*} w\right)$. On notera $2 g$ la dimension de $\mathfrak{V}$ sur $\mathbb{Q}$.

On fixe dans toute la suite un nombre premier $p$, et on fera l'hypothèse que $p>2 g$. Le rôle de cette hypothèse est expliqué au paragraphe 3.3.1.

On suppose que $B$ est non ramifié en $p$, c'est-à-dire que $B_{\mathbb{Q}_{p}}$ est un produit d'algèbres de matrices sur des extensions non-ramifiées de $\mathbb{Q}_{p}$.

On se donne un $\mathbb{Z}_{(p)}$-ordre $\mathcal{O}_{B}$ dans $B$ qui devient un ordre maximal de $B_{\mathbb{Q}_{p}}$ après tensorisation par $\mathbb{Z}_{p}$, et stable par l'involution de $B$.

On se donne aussi $\mathcal{V}$ un $\mathcal{O}_{B}$-réseau de $\mathfrak{V}$ autodual. Le fait que $\mathcal{V}$ soit autodual implique en particulier que la forme bilinéaire induite sur $\mathcal{V}$ est non dégénérée.

Soit $C$ l'anneau des endomorphismes $B$-linéaires de $\mathfrak{V}$.

On définit le groupe $G$ par $G(R)=\left\{g \in(C \otimes R)^{*}, \exists \mu \in R^{*}, \forall v, w \in \mathfrak{V} \otimes\right.$ $R,(g v, g w)=\mu(v, w)\}$, pour toute $\mathbb{Z}_{(p)}$-algèbre $R$.

On se donne un $\mathbb{R}$-homomorphisme d'algèbres $h: \mathbb{C} \rightarrow C_{\infty}=C \otimes_{\mathbb{Q}} \mathbb{R}$ tel que $h(z)^{*}=h(\bar{z})$, et la forme $(v, h(i) w)$ soit définie positive sur $\mathfrak{V}_{\infty}=\mathfrak{V} \otimes_{\mathbb{Q}} \mathbb{R}$. On associe à $h$ le morphisme $\mu_{h}: \mathbb{C}^{*} \rightarrow G_{\mathbb{C}}$, qui définit la filtration de Hodge sur $\mathfrak{V}_{\mathbb{C}}$, c'est-à-dire la décomposition $\mathfrak{V}=\mathfrak{V}_{z} \oplus \mathfrak{V}_{1}$, où $\mu_{h}(z)$ agit par $z$ sur $\mathfrak{V}_{z}$ et par 1 sur $\mathfrak{V}_{1}$.

Le corps dual associé à ces données est le corps $E(G, h)$ qui est le corps de définition de la classe d'isomorphisme de $\mathfrak{V}_{z}$ comme $B$-représentation. C'est le sous-corps de $\mathbb{C}$ engendré par les $\operatorname{tr}(b), b \in B$ agissant sur $\mathfrak{V}_{z}$.

\subsubsection{Deux cas particuliers}

Dans la suite nous nous intéresserons uniquement à deux cas particuliers : le cas Siegel et le cas unitaire. Le cas Siegel correspond à la situation où $B$ est réduit à $\mathbb{Q}$. La variété de Shimura associée est alors la variété modulaire de Siegel. Le cas unitaire correspond au cas où $B$ est une extension quadratique imaginaire de $\mathbb{Q}$. La forme alternée (, ) est alors la partie imaginaire d'une forme hermitienne sur $\mathfrak{V}$, qu'on peut voir comme un $B$-espace vectoriel de dimension moitié.

\subsubsection{Le problème de modules}

On peut associer aux données de Shimura précédentes un problème de modules, tel que décrit dans [10], dont on rappelle ici l'essentiel. 
Fixons $\mathcal{K}^{p}$ un sous-groupe compact ouvert de $G\left(\mathbb{A}_{f}^{p}\right)$. On considère le foncteur des $\mathcal{O}_{E(G, h)} \otimes \mathbb{Z}_{(p)}$-schémas dans les ensembles, qui à $S$ associe l'ensemble à équivalence près des quadruplets $(A, \lambda, i, \eta)$, où $A$ est un schéma abélien $A$ sur $S$, muni d'une polarisation $\lambda$ première à $p$, et d'une flèche $i: \mathcal{O}_{B} \rightarrow \operatorname{End}(A) \otimes \mathbb{Z}_{(p)}$ qui est un morphisme d'algèbres à involution, l'involution sur $\operatorname{End}(A) \otimes \mathbb{Z}_{(p)}$ étant l'involution de Rosati donnée par $\lambda$, et $\eta$ est une structure de niveau. Enfin on suppose que $\mathcal{O}_{B}$ agit sur $\operatorname{Lie}(A)$ comme sur $\mathfrak{V}_{z}$, c'est-à-dire que $\operatorname{det}(b, \operatorname{Lie}(A))=\operatorname{det}\left(b, \mathfrak{V}_{z}\right)$ pour tout $b \in \mathcal{O}_{B}$. La structure de niveau consiste en ce qui suit : on considère le $\mathbb{A}_{f}^{p}$-module de Tate de $A$, c'est un $\mathbb{A}_{f}^{p}$-faisceau lisse sur $S$. Soit $s$ un point géométrique de $S$, une structure de niveau consiste en une $\mathcal{K}^{p}$-orbite $\eta$ d'isomorphismes $\mathfrak{V}_{\mathbb{A}_{f}^{p}} \rightarrow H_{1}\left(A_{s}, \mathbb{A}_{f}^{p}\right)$ de $B$-modules munis d'une forme alternée, et qui soit fixée par $\pi_{1}(S, s)$.

Deux quadruplets $(A, \lambda, i, \eta)$ et $\left(A^{\prime}, \lambda^{\prime}, i^{\prime}, \eta^{\prime}\right)$ sont dit équivalents s'il existe une isogénie première à $p$ de $A$ vers $A^{\prime}$, commutant à l'action de $\mathcal{O}_{B}$, transformant $\eta$ en $\eta^{\prime}$, et $\lambda$ en un multiple scalaire $\left(\operatorname{dans} \mathbb{Z}_{(p)}^{*}\right)$ de $\lambda^{\prime}$.

Ce foncteur est représentable, par un schéma quasi-projectif et lisse $\mathcal{M}$ sur $\mathcal{O}_{E} \otimes \mathbb{Z}_{(p)}$ pourvu que l'on choisisse $\mathcal{K}^{p}$ suffisamment petit.

Notons $\mathcal{A}$ le schéma abélien universel sur $\mathcal{M}$.

Pour les cas Siegel et unitaire, on a le résultat suivant (lemme 7.2 de [10]) :

LEMME 1.1. - $\mathcal{O}_{\mathcal{M}} \otimes \mathcal{V}$ et $\mathcal{H}^{1}(\mathcal{A} / \mathcal{M})^{\vee}$ sont localement isomorphes comme $\mathcal{O}_{B}$-modules munis d'une forme alternée.

\subsubsection{La situation géométrique considérée}

On obtient alors la situation suivante : Notons $K$ le complété en une place $v \mid p$ de $E(G, h)$, et $\mathcal{O}$ son anneau des entiers. Notons $\mathcal{O}_{n}=\mathcal{O} / \varpi^{n+1}$, où $\varpi$ est une uniformisante de $\mathcal{O}$. C'est l'anneau des vecteurs de Witt de longueur $n$ sur le corps résiduel de $\mathcal{O}$ puisque $p$ est non ramifié dans $E(G, h)$. Posons $S=\operatorname{Spec} \mathcal{O}$, et $S_{n}=\operatorname{Spec} \mathcal{O}_{n}$. D'une façon générale, on notera avec un indice $n$ la réduction d'un $\mathcal{O}$-schéma modulo $\varpi^{n+1}$.

On notera $X=\mathcal{M}_{\mathcal{O}}$, c'est donc un schéma lisse sur $S$, muni d'un schéma abélien $\mathcal{A}$, provenant du schéma abélien universel sur la variété de Shimura. De plus, on a un morphisme $\mathcal{O}_{B} \rightarrow \operatorname{End}(A) \otimes_{\mathbb{Z}} \mathbb{Z}_{(p)}$. On note $f: \mathcal{A} \rightarrow X$, et $f_{s}: \mathcal{A}^{s} \rightarrow X$ les morphismes structuraux, où $\mathcal{A}^{s}$ est le produit fibré de $\mathcal{A}$ avec lui-même au-dessus de $X s$ fois.

\subsection{Existence de compactifications}

Nous aurons besoin d'utiliser aussi des compactifications de $X$, ainsi que du schéma abélien universel $\mathcal{A}$ et de ses puissances. Nous résumons dans ce paragraphe les seules propriétés de ces compactifications que nous utilisons. 


\subsubsection{Compactifications de la base}

Nous avons besoin tout d'abord de compactification du modèle entier de la variété de Shimura. Dans le cas qui nous intéresse, celui des variétés de Shimura de type PEL ayant bonne réduction, nous disposons grâce à la technique des compactifications toroïdales du résultat suivant (démontré par Kai-Wen Lan dans sa thèse [11], qui généralise le cas des variétés de Siegel traité dans [4]) :

Proposition 1.2. - Il existe un schéma $\bar{X}$ propre et lisse sur $S$, contenant $X$ comme ouvert dense, tel que le complémentaire de $X$ dans $\bar{X}$ est un diviseur à croisements normaux relatifs.

On fixera dans la suite une fois pour toute une telle compactification $\bar{X}$. On peut remarquer que les résultats ne dépendent en fait pas du choix de $\bar{X}$ parmi l'ensemble des compactifications torö̈dales : deux compactifications torö̈dales sont toujours comparables, au sens où il existe une troisième qui les domine toutes les deux, ce qui permet de voir que les groupes de cohomologie décrits en 4.2.5 ne dépendent pas de ce choix de compactification.

\subsubsection{Compactifications du schéma abélien universel}

Nous avons fixé dans le paragraphe précédent un schéma $\bar{X}$ propre lisse sur $S$, muni d'un diviseur à croisements normaux relatifs $D$, contenant $X$ comme ouvert dense. Pour chaque entier $s \geq 1$, on appelle bonne compactification de $\mathcal{A}^{s}$ un schéma $\overline{\mathcal{A}^{s}}$ propre et lisse sur $\bar{X}$, dont la restriction à $X$ est $\mathcal{A}^{s}$, tel que $f_{s}^{-1}(\bar{X} \backslash X)$ est un diviseur à croisements normaux relatifs.

Nous ferons dans toute la suite l'hypothèse suivante :

Hypothèse 1. - Il existe pour tout $s \geq 1$ une famille de bonnes compactifications de $\mathcal{A}^{s}$ vérifiant les propriétés suivantes:

1. Pour toute isogénie $u$ de $\mathcal{A}^{s}$, il existe deux bonnes compactifications $\overline{\mathcal{A}}_{1}$ et $\overline{\mathcal{A}^{s}}$ 2 de $\mathcal{A}^{s}$, et un $\bar{X}$-morphisme $\overline{\mathcal{A}^{s}}{ }_{1} \rightarrow \overline{\mathcal{A}^{s}}$ 2 prolongeant $u$.

2. Étant donné deux bonnes compactifications $\overline{\mathcal{A}}^{s}$ et $\overline{\mathcal{A}}^{s}$ de $\mathcal{A}^{s}$, il en existe une troisième ${\overline{\mathcal{A}^{s}}}_{3}$ et des $\bar{X}$-morphismes ${\overline{\mathcal{A}^{s}}}_{3} \rightarrow{\overline{\mathcal{A}^{s}}}_{1}$ et ${\overline{\mathcal{A}^{s}}}_{3} \rightarrow \overline{\mathcal{A}^{s}}$ induisant l'identité sur $\mathcal{A}^{s}$.

Nous fixerons dans toute la suite une telle famille de compactifications.

Lorsque la compactification $\bar{X}$ peut être obtenue en rajoutant à $X$ un diviseur lisse, on sait que l'hypothèse 1 est vérifiée : c'est l'objet de l'article [14]. C'est une situation qui est obtenue en particulier dans le cas où le groupe $G$ considéré est un groupe unitaire du type $\mathrm{GU}(n, 1)$.

L'hypothèse est vérifiée aussi dans le cas des variétés de Siegel : l'existence d'une telle famille se déduit des résultats du livre [4, chapitre VI], où sont construites des compactifications toroïdales $\operatorname{des} \mathcal{A}^{s}$.

TOME $137-2009-\mathrm{N}^{\mathrm{O}} 3$ 
Dans le cas PEL général, on ne dispose pas d'une preuve de cette hypothèse. Il est toutefois probable que les techniques de compactifications toroïdales décrites dans [4] se généralisent à la situation étudiée dans [11], et que l'hypothèse soit donc vérifiée.

Dans le cas Siegel, nous aurons besoin d'une propriété supplémentaire de la famille de compactifications :

Hypothèse 2. - La famille de compactifications vérifie de plus la propriété suivante : si $\mathcal{L}$ est un faisceau symétrique sur $\mathcal{A}^{s}$, il existe des entiers $a$ et $b$, et une compactification de la famille $\overline{\mathcal{A}^{s}}$ tels que le faisceau $\left(\mathcal{O}(2)^{\otimes a} \otimes \mathcal{L}\right)^{\otimes b}$ se prolonge en un faisceau sur $\overline{\mathcal{A}^{s}}$. De plus, on peut choisir $a$ et $b$ premiers à $p$.

Cette construction fait l'objet du chapitre VI du livre [4]. Elle n'est détaillée que pour le cas où le faisceau symétrique ample considéré est $\mathcal{O}(2)$, mais cela s'adapte au cas d'un faisceau symétrique ample quelconque, pour lequel on prendra donc $\mathcal{O}(2)^{\otimes a} \otimes \mathcal{L}$, avec $a$ assez grand pour que le faisceau soit ample.

\section{Représentations de $G$}

L'objet de cette partie est de dégager la notion de représentation atteignable de $G$. Ce sont les représentations qui donnent des faisceaux dont la cohomologie peut être découpée par des correspondances algébriques dans la cohomologie de la variété abélienne universelle, et pour lesquels nous pourrons obtenir des théorèmes de comparaison sur la cohomologie.

Les objets de la catégorie $\operatorname{Rep}^{a}(G)$ ainsi définie étant les seules représentations de $G$ pour lesquelles nous pourrons obtenir un théorème de comparaison, il est important de les comprendre et en particulier de vérifier que l'on en obtient une quantité suffisante. Nous nous intéresserons ici à deux cas particuliers : nous décrivons dans le paragraphe 2.3 quelles sont les représentations atteignables dans le cas où le groupe $G$ est un groupe unitaire, et dans le paragraphe 2.4 dans le cas où le groupe est le groupe symplectique. Il serait intéressant d'avoir une description de cette catégorie dans le cas d'autres groupes, par exemple les cas correspondant aux variétés de Hilbert-Blumenthal.

On note $\operatorname{Rep}(G)$ la catégorie des représentations de $G$ sur un $\mathbb{Z}_{(p)}$-module libre de type fini, et $\operatorname{Rep}(G)_{\mathbb{Q}}$ celles des représentations de $G$ sur un $\mathbb{Q}$-espace vectoriel de dimension finie.

On note $\mathcal{V}_{0} \in \operatorname{Rep}(G)$ la duale de la représentation standard de $G$, c'est-àdire la duale du réseau $\mathcal{V}$ défini au paragraphe 1.1.1. 


\subsection{Représentations atteignables}

\subsubsection{Les algèbres d'endomorphismes}

Les représentations de $G$ de la forme $\wedge^{\bullet} \mathcal{V}_{0}^{s}$, pour $s \geq 1$, jouent un rôle particulier : les faisceaux que nous allons leur associer dans la section 3 ont une interprétation géométrique. Nous définissons deux sous-algèbres de l'algèbre $\operatorname{End}\left(\wedge^{\bullet} \mathcal{V}_{0}^{s}\right)$ des endomorphismes $G$-linéaires de $\wedge^{\bullet} \mathcal{V}_{0}^{s}$, formées de morphismes ayant aussi une interprétation géométrique qui sera précisée dans le paragraphe 3.2.1. L'objectif est de pouvoir découper dans les $\wedge^{\bullet} \mathcal{V}_{0}^{s}$ des représentations irréductibles de $G$ à l'aide de cette algèbre d'endomorphismes, en s'inspirant des constructions de Weyl.

Commençons par définir pour tout $s \geq 1$ une sous- $\mathbb{Z}_{(p)}$-algèbre $E(A)_{s}$ de $\operatorname{End}\left(\wedge^{\bullet} \mathcal{V}_{0}^{s}\right)$. C'est l'algèbre engendrée par l'action de $\mathrm{M}_{s}(\mathbb{Z})$ sur $\mathcal{V}_{0}^{s}$, muni de la multiplication opposée, et par l'action de $\mathcal{O}_{B}$ sur $\mathcal{V}_{0}$.

Définissons ensuite l'algèbre $E(C)_{s}$. On note $\mathbb{Z}_{(p)}(1)$ la représentation du groupe $G$ correspondant à l'action du groupe sur $\mathbb{Z}_{(p)}$ par le multiplicateur. Observons que $\mathcal{V}_{0}=\mathcal{V}(-1)$.

On note $u \in \wedge^{2} \mathcal{V}_{0}^{2}(1)$ l'élément provenant de la forme bilinéaire sur $\mathfrak{V}$, et pour tous $1 \leq i<j \leq s$, on note $u_{i, j}$ l'image de $u$ par l'application $\wedge^{2} \mathcal{V}_{0}^{2}(1) \rightarrow$ $\wedge^{2} \mathcal{V}_{0}^{s}(1)$ induite par l'application $\mathcal{V}_{0}^{2} \rightarrow \mathcal{V}_{0}^{s}$ consistant à placer les deux facteurs $\mathcal{V}_{0}$ aux places $i$ et $j$. Le cup-produit par $u_{i, j}$ définit une application $\varphi_{i, j}$ : $\wedge^{\bullet} \mathcal{V}_{0}^{s}(-1) \rightarrow \wedge^{\bullet} \mathcal{V}_{0}^{s}$ qui envoie chaque $\wedge^{k} \mathcal{V}_{0}^{s}(-1)$ dans $\wedge^{k+2} \mathcal{V}_{0}^{s}$. L'application duale de $\varphi_{i, j}$ permet de définir une application $\psi_{i, j}: \wedge^{\bullet} \mathcal{V}_{0}^{s} \rightarrow \wedge^{\bullet} \mathcal{V}_{0}^{s}(-1)$. Enfin on note $\theta_{i, j}=\varphi_{i, j} \circ \psi_{i, j}$, qui est donc un endomorphisme de $\wedge^{\bullet} \mathcal{V}_{0}^{s}$.

On définit alors $E(C)_{s}$ comme la sous- $\mathbb{Z}_{(p)}$-algèbre de $\operatorname{End}\left(\wedge^{\bullet} \mathcal{V}_{0}^{s}\right)$ engendrée par l'action de $\mathrm{M}_{s}(\mathbb{Z})$ et les $\theta_{i, j}, 1 \leq i<j \leq s$.

On notera $E_{s}$ pour désigner indifféremment $E(A)_{s}$ et $E(C)_{s}$. On dira que $u \in E_{s}$ est un projecteur homogène (de degré $t$ ) si son image est contenue dans $\wedge^{t} \mathcal{V}_{0}^{s} \subset \wedge^{\bullet} \mathcal{V}_{0}^{s}$.

On donne des définitions similaires pour les éléments de $E_{s} \otimes \mathbb{Q}$ agissant sur $\wedge^{\bullet}\left(\mathcal{V}_{0} \otimes \mathbb{Q}\right)^{s}$.

\subsubsection{Définition des sous-catégories}

DÉFInition 2.1. - On note $\operatorname{Rep}^{a}(G)$ la sous-catégorie de $\operatorname{Rep}(G)$ formée des représentations isomorphes à une somme directe de représentations de $G$ de la forme $\operatorname{im} q$, où $q$ est un projecteur homogène de $E_{s}$ agissant sur un $\wedge^{\bullet} \mathcal{V}_{0}^{s}$. Si $V \in \operatorname{Rep}^{a}(G)$, on note $t(V)$ le plus grand degré des projecteurs homogènes qui apparaissent dans la définition de $V$. Lorsque on a besoin de préciser s'il s'agit de $E(A)$ ou $E(C)$, on note $\operatorname{Rep}_{A}^{a}(G)$ et $\operatorname{Rep}_{C}^{a}(G)$ respectivement.

On définit de façon similaire $\operatorname{Rep}^{a}(G)_{\mathbb{Q}}$.

TOME $137-2009-\mathrm{N}^{\mathrm{O}} 3$ 
Comme nous n'obtenons des résultats que pour les représentations de $G$ qui sont dans $\operatorname{Rep}^{a}(G)$, il va s'agir de voir que cette sous-catégorie n'est pas trop petite, et qu'il n'est donc pas trop restrictif de s'y limiter. C'est l'objet des paragraphes suivants.

\subsection{Poids $p$-petits}

Nous introduisons la notion de poids $p$-petit, qui joue un rôle dans la description des représentations qui sont dans $\operatorname{Rep}^{a}(G)$.

Supposons notre groupe réductif $G$ déployé sur un certain corps $E$. Les représentations irréductibles de $G$ sur $E$ sont paramétrées par l'ensemble des poids dominants, une fois fixé un tore maximal et un système de racines positives. Si $a$ est un poids dominant, on note $V_{E}(a)$ la représentation irréductible de $G$ sur $E$ de plus haut poids $a$.

On définit comme dans [9, II.3.15], ce qu'est un poids dominant p-petit. La propriété qui nous intéresse ici est la propriété suivante (voir $[13,1.9]$ ) : si le poids $a$ est $p$-petit, alors il existe à homothétie près un unique réseau dans $V_{E}(a)$ qui est stable sous l'action de $\operatorname{Dist}(G)$, l'algèbre des distributions de $G$ sur $\mathcal{O}_{E,(v)}$, pour $v$ une place de $E$ divisant $p$. Cela a donc un sens de définir $V(a)$ comme la représentation irréductible de $G$ sur $\mathcal{O}_{E,(v)}$ de plus haut poids $a$.

\subsection{Description de $\operatorname{Rep}^{a}(G)$ dans le cas unitaire}

Nous obtenons dans cette section une description partielle de la catégorie $\operatorname{Rep}^{a}(G)$ dans le cas unitaire : dans la proposition 2.6 nous indiquons un certain nombre de représentations qui apparaissent dans cette catégorie, mais nous n'en obtenons pas une description exhaustive.

Nous nous plaçons maintenant dans le cas où le groupe $G$ est un groupe unitaire, relatif à un corps $E$ quadratique imaginaire, qui correspond à l'algèbre $B$ du paragraphe 1.1.1, donc $G$ est de la forme $\mathrm{GU}\left(g_{1}, g_{2}\right)$, et il est déployé sur $E$. On a donc $G_{E} \stackrel{\sim}{\longrightarrow} \mathrm{GL}(g)_{E} \times \mathbb{G}_{m, E}$, où $g=g_{1}+g_{2}$.

L'ensemble des représentations sur $E$ irréductibles de $G$ est paramétré par les $g+1$-uplets $\left(a_{1}, \ldots, a_{g} ; c\right)$ d'entiers, avec $a_{1} \geq \cdots \geq a_{g}$ et $\sum a_{i}=c(\bmod 2)$, une fois choisi un isomorphisme entre $G_{E}$ et $\operatorname{GL}(g)_{E} \times \mathbb{G}_{m, E}$. Notons $\alpha$ et $\beta$ les deux morphismes de $E$ dans $E$, le choix de l'isomorphisme revient à en privilégier un des deux.

La représentation $\mathcal{V}_{0} \otimes E$ correspond à la somme de deux représentations irréductibles $V_{1}$ et $V_{2}, V_{1}$ de plus haut poids $(1,0, \ldots, 0 ; 1)$ et $V_{2}$ de plus haut poids $(0, \ldots, 0,-1 ; 1) . V_{1}$ est l'espace propre associé à la valeur propre $\alpha(x)$ de l'endomorphisme $u(x)$, pour tout $x$ dans $E$, et $V_{2}$ est l'espace propre associé à la valeur propre $\beta(x)$. 


\subsubsection{Description de $\operatorname{Rep}^{a}(G)_{\mathbb{Q}}$}

Notons $\operatorname{Rep}^{a}(G)_{E}$ l'ensemble des $V \otimes_{\mathbb{Q}} E$, où $V \in \operatorname{Rep}^{a}(G)_{\mathbb{Q}}$, et $V_{0}=\mathcal{V}_{0} \otimes E$.

Proposition 2.2. - La catégorie $\operatorname{Rep}^{a}(G)_{E}$ contient toutes les représentations qui sont de la forme $V(a) \oplus V\left(a^{*}\right)$, où $V(a)$ est la représentation irréductible de plus haut poids $\left(a_{1}, \ldots, a_{g} ; c\right)$ avec $a_{g} \geq 0$ et $c=\sum a_{i}=s$, et $a^{*}$ est le poids $\left(-a_{g}, \ldots,-a_{1} ; c\right)$.

Afin de démontrer cette proposition, nous établissons quelques lemmes préliminaires.

Lemme 2.3. - Si $a=\left(a_{1}, \ldots, a_{g} ; c\right)$ est un poids dominant de $G$, tel que $a_{g} \geq 0$ et $c=\sum a_{i}$ et $V(a)$ est la représentation irréductible associée, alors $i l$ existe un élément $C_{a}$ de $\mathbb{Q S}_{s}$ tel que $V(a)=C_{a} V_{1}^{\otimes s}$ et $V\left(a^{*}\right)=C_{a} V_{2}^{\otimes s}$, où $s=\sum a_{i}$, et $\mathfrak{S}_{s}$ est le groupe des permutations.

Démonstration. - Regardons d'abord $V(a)$ comme une représentation de $\mathrm{GL}_{g}$, en oubliant l'action du multiplicateur. Comme expliqué dans [6], 15.5, il existe un $C_{a} \in \mathbb{Q} \mathfrak{S}_{s}$ idempotent tel que $V(a)=C_{a} V_{1}^{\otimes s}, V(a)$ et $V_{1}$ étant vues toutes deux comme des représentations de $\mathrm{GL}_{g}$. Il faut voir ensuite que l'égalité tient aussi comme représentations de $\mathrm{GL}(g)_{E} \times \mathbb{G}_{m, E}$, donc que le multiplicateur agit de la même façon sur les deux. Or il agit par $x \mapsto x^{c}$ sur $V(a)$, et par $x \mapsto x^{s}$ sur $V_{1}^{\otimes s}$, et on a $s=c$.

Lemme 2.4. - Soit $s \geq 0$. Il existe un projecteur $q$ dans $E(A)_{s} \otimes \mathbb{Q}$ commutant à l'action de $\mathfrak{S}_{s}$ tel que l'image de q agissant sur $\wedge \wedge^{\bullet} V_{0}^{s}$ est $V_{0}^{\otimes s}$.

Démonstration. - On a $\wedge^{\bullet} V_{0}^{s}=\oplus_{0 \leq i_{1} \leq 2 g, \ldots, 0 \leq i_{s} \leq 2 g} \wedge^{i_{1}} V_{0} \times \cdots \times \wedge^{i_{s}} V_{0}$.

Considérons un entier $m$ non nul, $v_{j}$ la matrice diagonale $[(1, \ldots, 1, m, 1, \ldots)]$ avec un $m$ en $j$-ème position. L'espace propre correspondant à la valeur propre $m$ est la somme des termes pour lesquels $i_{j}=1$. Soit $p_{j}$ le projecteur sur cet espace propre. Les $p_{j}$ commutent, leur produit est donc un projecteur $q$ sur l'intersection des images, c'est-à-dire les termes pour lesquels chaque $i_{j}$ est égal à 1 , c'est-à-dire $V_{0}^{\otimes s}$. De plus, $q$ commute bien à l'action de $\mathfrak{S}_{s}$.

Enfin on utilise que $V_{0}=V_{1} \oplus V_{2}, V_{0}^{\otimes s}$ est donc égal à une somme de termes de la forme $V_{1}^{\otimes x} \otimes V_{2}^{\otimes y}$ avec $x+y=s$.

LEMme 2.5. - Il existe un élément $q^{\prime}$ dans le centre de $E(A)_{s} \otimes \mathbb{Q}$ dont la restriction de l'action $\grave{a} V_{0}^{\otimes s}$ est un projecteur sur $V_{1}^{\otimes s} \oplus V_{2}^{\otimes s}$. 
Démonstration. - Fixons $z \in \mathcal{O}_{E} . z$ agit par $\alpha(z)$ sur $V_{1}$ et par $\beta(z)$ sur $V_{2}$. Notons $\alpha(z)=a$ et $\beta(z)=b$. Choisissons $z$ de sorte que les $a^{x} b^{y}$ soient tous distincts. Alors $V_{1}^{\otimes x} \otimes V_{2}^{\otimes y}$ est (dans $V_{0}^{\otimes s}$ ) l'espace propre associé à la valeur propre $a^{x} b^{y}$.

Soit $P$ le polynôme $\Pi_{r+t=s}\left(X-a^{r} b^{t}\right)$. Il est à coefficients entiers, et c'est le polynôme minimal de l'action de $u(z)$ sur $V_{0}^{\otimes s}$. Soit $Q=\Pi_{r+t=s, r \neq 0, t \neq 0}(X-$ $\left.a^{r} b^{t}\right)$. Alors $P=Q T$ où $T=\left(X-a^{s}\right)\left(X-b^{s}\right)$, et $Q$ et $T$ sont premiers entre eux. Il existe donc des polynômes $U$ et $V$ (à coefficients rationnels), tels que $U Q+V T=1$. Alors l'action de $u(1-U Q)(z)$ sur $V_{0}^{\otimes s}$ est un projecteur sur $V_{1}^{\otimes s} \oplus V_{2}^{\otimes s}$, qu'on note $q^{\prime}$.

Démonstration de la proposition 2.2. - Soit a comme dans l'énoncé, et $s=$ $c=\sum a_{i}$. Posons $P=C_{a} q^{\prime} q$, alors $P \wedge \bullet V_{0}^{s}$ est la représentation $V(a) \oplus V\left(a^{*}\right)$. En effet, notons que $C_{a}, q^{\prime}$ et $q$ commutent par construction, donc $P$ est un projecteur. On a $q \wedge^{\bullet} V_{0}^{s}=V_{0}^{\otimes s}, q^{\prime} q \wedge^{\bullet} V_{0}^{s}=V_{1}^{\otimes s} \oplus V_{2}^{\otimes s}, C_{a} q^{\prime} q \wedge^{\bullet} V_{0}^{s}=$ $C_{a} V_{1}^{\otimes s} \oplus C_{a} V_{2}^{\otimes s}$. Or $C_{a} V_{1}^{\otimes s}=V(a)$, et $C_{a} V_{2}^{\otimes s}=V\left(a^{*}\right)$.

Une fois décrites les représentations qui sont dans $\operatorname{Rep}^{a}(G)_{E}$, il faut maintenant retrouver quelle est la $\mathbb{Q}$-forme de ces représentations qui est dans $\operatorname{Rep}^{a}(G)_{\mathbb{Q}}$. $V_{1}$ et $V_{2}$ sont naturellement isomorphes, et $\operatorname{Gal}(E / \mathbb{Q})$ agit sur $V_{0}=V_{1} \oplus V_{2}$ par $(x, y) \mapsto(\bar{y}, \bar{x})$. Son action sur $V(a) \oplus V\left(a^{*}\right)$ peut être décrite par la même formule, ce qui nous permet d'obtenir les représentations qui sont dans $\operatorname{Rep}^{a}(G)_{\mathbb{Q}}$.

\subsubsection{Description de $\operatorname{Rep}^{a}(G)$}

Nous passons à présent à la description de $\operatorname{Rep}^{a}(G)$. Rappelons qu'au paragraphe précédent, nous avons défini une action $\operatorname{de} \operatorname{Gal}(E / \mathbb{Q}) \operatorname{sur} V(a) \oplus V\left(a^{*}\right)$.

Proposition 2.6. - La catégorie $\operatorname{Rep}^{a}(G)$ contient toutes les représentations qui sont de la forme $\left(V(a) \oplus V\left(a^{*}\right)\right)^{\operatorname{Gal}(E / \mathbb{Q})}$, où $V(a)$ est la représentation irréductible de plus haut poids $\left(a_{1}, \ldots, a_{g} ; c\right)$ avec $a_{g} \geq 0$ et $c=\sum a_{i}=s$, et $a^{*}$ est le poids $\left(-a_{g}, \ldots,-a_{1} ; c\right)$, a et $a^{*}$ sont $p$-petits, et $2 g<p$.

Il suffit de voir que si les conditions données sont vérifiées, on peut prendre des dénominateurs premiers à $p$ dans les lemmes du paragraphe 2.3.1 ce qui fait l'objet des trois lemmes ci-dessous.

Lemme 2.7. - On se place comme dans le lemme 2.3. Alors si $\sum a_{i}<p, C_{a}$ est dans $\mathbb{Z}_{(p)} \mathfrak{S}_{s}$.

Démonstration. $-C_{a}$ est de la forme $(1 / n) C_{a}^{\prime}$, où $C_{a}^{\prime}$ est dans $\mathbb{Z} \mathfrak{S}_{s}$, et $n$ est l'entier tel que $C_{a}^{\prime 2}=n C_{a}^{\prime}$. Or $n$ divise $s$ ! (voir $[6,4.2]$ ), donc $1 / n \in \mathbb{Z}_{(p)}$ si $s<p$. 
Lemme 2.8. - Dans le cadre du lemme 2.4, on peut choisir $q$ dans $E(A)_{s}$ dès que $p>2 g$.

Démonstration. - Lorsque on écrit $p_{j}$ comme un polynôme en $v_{j}$, les dénominateurs qui apparaissent sont les différences entre les valeurs propres de $v_{j}$, qui sont les $m^{i}$ pour $0 \leq i \leq 2 g$. Si $2 g<p$, on peut prendre un $m$ dont l'image dans $\mathbb{Z} / p \mathbb{Z}$ est un générateur de $\mathbb{Z} / p \mathbb{Z}^{*}$, de sorte que les $m^{i}-m^{i^{\prime}}$ sont tous premiers à $p$.

Lemme 2.9. - Dans le lemme 2.5, on peut prendre $q^{\prime}$ dans $E(A)_{s}$ dès que $p>s$.

Démonstration. - Écrivons donc $U Q+V T=c$, avec $U$ et $V$ à coefficients entiers et $c$ entiers, et étudions les facteurs premiers de $c$. On obtient $c=$ $Q\left(a^{s}\right) Q\left(b^{s}\right)$. Il s'agit donc de trouver $z \in \mathcal{O}_{E}$ tel que $c$ soit premier à $p$ (et que aucun des $a^{r} b^{t}, r>0, t>0, r+t=s$ ne soit égal à $a^{s}$ ou à $\left.b^{s}\right)$.

On a $Q\left(a^{s}\right)=a^{s(s-1) / 2} \Pi_{1 \leq t \leq s-1}\left(a^{t}-b^{t}\right)$, et $Q\left(b^{s}\right)=b^{s(s-1) / 2} \Pi_{1 \leq t \leq s-1}\left(b^{t}-\right.$ $\left.a^{t}\right)$. Supposons d'abord que $p$ est inerte dans $E$. Alors $\mathcal{O}_{E} / p$ est isomorphe à $\mathbb{F}_{p^{2}}$. La conjugaison dans $\mathcal{O}_{E}$ se traduit par $x \mapsto x^{p}$ dans $\mathcal{O}_{E} / p$. Choisissons donc $x$ un générateur de $\mathbb{F}_{p^{2}}^{*}$, alors un $z$ relevant $x$ convient.

Supposons maintenant $p$ décomposé dans $E$. Alors $\mathcal{O}_{E} / p$ est égal à $\mathbb{F}_{p} \times \mathbb{F}_{p}$, et la conjugaison dans $\mathcal{O}_{E}$ échange les deux facteurs dans $\mathcal{O}_{E} / p$. Choisissons un $x$ dans $\mathbb{F}_{p}^{*}$ tel que $x^{i} \neq 1$ pour tout $i$ entre 1 et $s$, et prenons $u$ et $v$ dans $\mathbb{F}_{p}^{*}$ tels que $u / v=x$. Alors si $z$ est un relèvement de $(u, v)$, il convient.

La proposition 2.6 est donc démontrée.

\subsection{Description de $\operatorname{Rep}^{a}(G)$ dans le cas Siegel}

Plaçons-nous maintenant dans le cas où $G=\mathrm{Gsp}_{2 g}$ est un groupe symplectique. On observe que $\operatorname{Rep}_{A}^{a}(G)$ est constitué des représentations de $\operatorname{Gsp}_{2 g}$ qui peuvent se prolonger en une représentation de $\mathrm{GL}_{2 g}$. Excepté dans le cas où $g=1$, cela ne constitue qu'une petite partie des représentations de $\mathrm{Gsp}_{2 g}$. Ceci justifie l'introduction de la catégorie $\operatorname{Rep}_{C}^{a}(G)$. On peut trouver dans l'article [12] une description des représentations qui figurent dans cette catégorie : on obtient, à l'action du centre près, toutes les représentations irréductibles dont le plus haut poids est $p$-petit.

TOME $137-2009-\mathrm{N}^{\circ} 3$ 


\section{Les faisceaux}

\subsection{Constructions fonctorielles}

\subsubsection{Cas étale}

Plaçons-nous dans la situation décrite au paragraphe 1.1.4. Soit $x$ un point géométrique de $X_{K}$. À chaque représentation du groupe fondamental de la variété $\pi_{1}\left(X_{K}, x\right)$ correspond un système local sur $X_{K}$. Considérons le faisceau constant $\mathbb{Z}_{p}$ sur $\mathcal{A}$, et $F=R^{1} f_{K *} \mathbb{Z}_{p}(1)$, où $f_{K}: \mathcal{A}_{K} \rightarrow X_{K}$ est le morphisme structural. $F$ correspond à la représentation standard de $G$ sur $\mathbb{Z}_{p}$, autrement dit à un morphisme $\pi_{1}\left(X_{K}, x\right) \rightarrow G\left(\mathbb{Z}_{p}\right)$. On peut donc associer par composition un système local à toute représentation sur $\mathbb{Z}_{p}$ de $G$, et ceci de façon fonctorielle. On note $\mathbb{F}(V)$ le système local associé à la représentation $V$. On définit de même le foncteur $\mathbb{F}_{n}(V)$, qui à $V$ associe un fibré en $\mathbb{Z} / p^{n} \mathbb{Z}$-modules, vérifiant $\mathbb{F}(V) \otimes_{\mathbb{Z}_{p}} \mathbb{Z} / p^{n} \mathbb{Z}=\mathbb{F}_{n}(V)$.

On observe que $\mathbb{F}\left(\mathcal{V}_{0}\right)=R^{1} f_{*} \mathbb{Z}_{p}$, et plus généralement $\mathbb{F}\left(\wedge^{t} \mathcal{V}_{0}^{s}\right)=$ $R^{t} f_{s, K *} \mathbb{Z}_{p}$, où $f_{s, K}$ est le morphisme structural $\mathcal{A}_{K}^{s} \rightarrow X_{K}$.

\subsubsection{Cas des fibrés à connexion}

Proposition 3.1. - Il existe un foncteur $\mathcal{F}$ de $\operatorname{Rep}(G)$ dans l'ensemble des $\mathcal{O}_{X}$-modules à connexion intégrable sur $X$, et pour tout $n$ un foncteur $\mathcal{F}_{n}$ de $\operatorname{Rep}(G)$ dans l'ensemble des $\mathcal{O}_{X_{n}}$-modules à connexion intégrable sur $X_{n}$, ces deux foncteurs étant compatibles.

Ici compatible, signifie que $\mathcal{F}_{n}\left(V / \varpi^{n+1}\right)=\mathcal{F}(V) / \varpi^{n+1}$. On ne va faire la construction que sur $\mathcal{O}_{X}$, la construction sur $\mathcal{O}_{X_{n}}$ s'obtenant par des méthodes similaires.

Notons $\mathcal{H}_{1}(\mathcal{A})=\left(R^{1} f_{*} \Omega_{\mathcal{A} / X}^{\bullet}\right)^{\vee}$. Introduisons $\mathcal{T}=\operatorname{Isom}\left(\mathcal{O}_{X} \otimes \mathcal{V}, \mathcal{H}_{1}(\mathcal{A})\right)$, les isomorphismes devant respecter la structure de $B$-module et la forme alternée à une constante près. C'est un torseur sur $X$ sous l'action (à droite) de $G$, en effet les deux faisceaux en question sont localement isomorphes, comme expliqué dans le lemme 1.1.

Soit $V \in \operatorname{Rep}(G)$, on note $\mathcal{F}(V)$ le faisceau des sections du fibré $\mathcal{T} \times{ }^{G} V$. C'est un faisceau de $\mathcal{O}_{X}$-modules quasi-cohérent. De même un morphisme entre représentations se transforme en morphisme entre faisceaux.

Ce fibré est muni d'une connexion qui provient de la connexion de GaussManin sur $\mathcal{H}_{1}(\mathcal{A})$.

Notons $\mathcal{H}^{i}\left(\mathcal{A}^{s}\right)=R^{i} f_{s *} \Omega_{\mathcal{A}^{s} / X}$, on a alors $\mathcal{F}\left(\wedge^{t} \mathcal{V}_{0}^{s}\right)=\mathcal{H}^{t}\left(\mathcal{A}^{s}\right)$. De même on notera $\mathcal{H}^{i}\left(\mathcal{A}_{n}^{s}\right)=R^{i} f_{s *} \Omega_{\mathcal{A}_{n}^{s} / X_{n}}$. 


\subsection{Action de $E_{s}$}

\subsubsection{Traduction géométrique}

Comme $E_{s}$ est une sous-algèbre de $\operatorname{End}\left(\wedge^{\bullet} \mathcal{V}_{0}^{s}\right)$, par fonctorialité de $\mathbb{F}$ et $\mathcal{F}$, on a donc aussi des morphismes de $\mathbb{Z}_{(p)}$-algèbres $E_{s} \stackrel{a_{t}}{\rightarrow} \operatorname{End}\left(R^{\bullet} f_{s, *} \mathbb{Z}_{p}\right)$ et $E_{s} \stackrel{a_{\text {cris }}}{\longrightarrow} \operatorname{End}\left(\mathcal{H}^{\bullet}\left(\mathcal{A}^{s}\right)\right)$. On va donner une interprétation géométrique de ces deux morphismes.

Soit $\mathcal{G} \subset E_{s}$ la partie formée des éléments suivants : les matrices de déterminant non nul, dans le cas unitaire les éléments non nuls de $\mathcal{O}_{B}$, dans le cas Siegel les opérations $\theta_{i, j}, 1 \leq i<j \leq s$ définies au paragraphe 2.1.1. L'ensemble $\mathcal{G}$, qu'on appellera ensemble des éléments géométriques de $E_{s}$, engendre $E_{s}$ comme $\mathbb{Z}_{(p)}$-algèbre.

Soit $u \in \mathcal{G}$ qui provient d'une matrice ou, dans le cas unitaire, d'un élément de $\mathcal{O}_{B}$. Alors $u$ provient d'un élément de $\operatorname{End}\left(\mathcal{V}_{0}^{s}\right)$, qui agit naturellement sur $\mathcal{A}^{s} / X$, donc sur $R^{\bullet} f_{s, *} \mathbb{Z}_{p}$ et $\mathcal{H}^{\bullet}\left(\mathcal{A}^{s}\right)$ par $a_{\mathrm{t}}(u)$ et $a_{\text {cris }}(u)$ respectivement.

Soit $\mathcal{P}$ le faisceau sur $\mathcal{A} \times \mathcal{A}$ provenant par la polarisation du faisceau de Poincaré sur $\mathcal{A} \times \mathcal{A}^{t}$. Pour $1 \leq i<j \leq s$ on note $\mathcal{P}_{i, j}$ le faisceau sur $\mathcal{A}^{s}$ obtenu en tirant $\mathcal{P}$ par le morphisme de projection sur les $i$-ièmes et $j$-ièmes facteurs $\mathcal{A}^{s} \rightarrow \mathcal{A} \times \mathcal{A}$. Alors l'opération consistant à faire le cup-produit par la première classe de Chern de $\mathcal{P}_{i, j}$ correspond à $a_{\mathrm{t}}\left(\psi_{i, j}\right)$ et $a_{\text {cris }}\left(\psi_{i, j}\right)$, l'opération duale correspond à $a_{\mathrm{t}}\left(\varphi_{i, j}\right)$ et $a_{\text {cris }}\left(\varphi_{i, j}\right)$, comme expliqué dans [12].

Par fonctorialité des constructions précédentes, on a, pour une représentation $V$ de la forme $V=q\left(\wedge^{\bullet} \mathcal{V}_{0}^{s}\right), q$ étant un projecteur de $E_{s}: \mathbb{F}(V)=$ $a_{\mathrm{t}}(q) R^{\bullet} f_{s, K *} \mathbb{Z}_{p}$, et $\mathcal{F}(V)=a_{\text {cris }}(q) \mathcal{H}^{\bullet}\left(\mathcal{A}^{s}\right)$.

On notera encore $a_{\text {cris }}$ et $a_{\mathrm{t}}$ les morphismes naturels de $E_{s}$ vers $\operatorname{End}\left(\mathcal{H}^{\bullet}\left(\mathcal{A}_{n}^{s}\right)\right)$ et $\operatorname{End}\left(R^{\bullet} f_{s *} \mathbb{Z} / p^{n} \mathbb{Z}\right)$ respectivement.

\subsubsection{Conséquence sur les faisceaux à connexion}

Lemme 3.2. - Pour tout $V \in \operatorname{Rep}^{a}(G)$ la connexion sur $\mathcal{F}(V)$ et sur $\mathcal{F}_{n}(V)$ est quasi-nilpotente.

Démonstration. - Notons que les opérations élémentaires commutent à la connexion sur $\mathcal{H}^{\bullet}\left(\mathcal{A}^{s}\right)$ induite par la connexion de Gauss-Manin, de sorte que, en reprenant les notations précédentes, $\mathcal{F}(V)$ est stable par la connexion de $\mathcal{H}^{\bullet}\left(\mathcal{A}^{s}\right)$. Comme la connexion de Gauss-Manin sur $\mathcal{H}^{\bullet}\left(\mathcal{A}^{s}\right)$ est quasi-nilpotente, c'est aussi le cas pour la connexion sur $\mathcal{F}(V)$.

Comme $X$ est lisse sur $S$, chaque $X_{n}$ est un relèvement de $X_{0}$ qui est lisse sur $S_{n}$. Les faisceaux $\mathcal{F}_{n}(V)$, qui sont des $\mathcal{O}_{X_{n}}$-modules cohérents munis d'une connexion intégrable et quasi-nilpotente, définissent donc des cristaux sur $\left(X_{0} / S_{n}\right)_{\text {cris }}$, ainsi que dans $\left(X_{m} / S_{n}\right)_{\text {cris }}$ pour tout $m \leq n$. On peut donc voir $\mathcal{F}_{n}$ 
comme un foncteur de $\operatorname{Rep}^{a}(G)$ vers la catégorie des cristaux $\operatorname{sur}\left(X_{0} / S_{n}\right)_{\text {cris }}$. Avec cette interprétation les $\mathcal{H}^{i}\left(\mathcal{A}_{n}^{s}\right)$ s'identifient aux $R^{i} f_{s, \text { cris* }} \mathcal{O}_{\mathcal{A}_{0}^{s} / S_{n}}$.

Lemme 3.3. - Pour tout $V \in \operatorname{Rep}^{a}(G)$, les faisceaux $\mathcal{F}(V)$ et $\mathcal{F}_{n}(V)$ sont localement libres sur $X$ et $X_{n}$ respectivement.

En effet c'est le cas pour les $\mathcal{H}^{n}\left(\mathcal{A}^{s}\right)$.

\subsection{Prolongement des cristaux}

L'objectif est de construire un foncteur $\overline{\mathcal{F}}$ de $\operatorname{Rep}^{a}(G)$ vers l'ensemble des fibrés localement libres sur $\bar{X}$ munis d'une connexion à pôles logarithmiques le long de $\bar{X} \backslash X$ intégrable et quasi-nilpotente, qui prolonge $\mathcal{F}$.

\subsubsection{Unicité du prolongement}

Lemme 3.4. - Soit $\mathcal{E}$ un fibré localement libre sur $X$ muni d'une connexion intégrable et quasi-nilpotente.

$S^{\prime}$ 'il existe un prolongement de $\mathcal{E}$ en un fibré localement libre sur $\bar{X}$ muni d'une connexion à pôles logarithmiques le long de $\bar{X} \backslash X$ intégrable et quasinilpotente, alors il est unique, et de plus tout prolongement de $\mathcal{E}$ en un fibré cohérent sur $\bar{X}$ muni d'une connexion ayant les mêmes propriétés est aussi localement libre (et donc égal au prolongement précédent).

De plus, si $\mathcal{E}_{1}$ et $\mathcal{E}_{2}$ sont deux tels faisceaux admettant des prolongements, et $u: \mathcal{E}_{1} \rightarrow \mathcal{E}_{2}$ est un morphisme horizontal, u admet un unique prolongement horizontal entre les prolongements des faisceaux.

Démonstration. - Soit $\overline{\mathcal{E}}$ un tel prolongement cohérent. Regardons tout d'abord $\mathcal{E}_{K}$. D'après [3], il existe au plus un prolongement de $\mathcal{E}_{K}$ en un fibré muni d'une connexion à pôles logarithmiques, qui est l'extension canonique de Deligne, ce prolongement est donc nécessairement $\overline{\mathcal{E}}_{K}$.

Le faisceau $\overline{\mathcal{E}}$ est alors uniquement déterminé. En effet, notons $X^{\prime}$ la réunion de $X$ et $\bar{X}_{K}$ dans $\bar{X}, j$ l'inclusion de $X^{\prime}$ dans $\bar{X}$, et $\mathcal{E}^{\prime}$ le faisceau qui coïncide avec $\mathcal{E}$ sur $X$ et avec $\overline{\mathcal{E}}_{K}$ sur $\bar{X}_{K}$. Alors pour des raisons de codimension, et le faisceau $\overline{\mathcal{E}}$ étant cohérent, $\overline{\mathcal{E}}=j_{*}\left(\mathcal{E}^{\prime}\right)$. En particulier, tous les prolongements cohérents munis de connexion sont égaux en tant que faisceaux, donc si l'un est localement libre, tous le sont.

Enfin, $\overline{\mathcal{E}}$ étant localement libre, sa connexion est entièrement déterminée par sa restriction à $\overline{\mathcal{E}}_{K}$.

Pour l'existence du prolongement des morphismes, cela provient de la fonctorialité de l'extension canonique de Deligne. 
Corollaire 3.5. - Le fibré $\mathcal{H}^{i}\left(\overline{\mathcal{A}^{s}}\right)$, muni de la connexion de Gauss-Manin, ne dépend pas du choix de la compactification $\overline{\mathcal{A}^{s}}$. De plus, étant données deux compactifications $\overline{\mathcal{A}}$ de $\mathcal{A}$ et $\overline{\mathcal{A}^{s}}$ de $\mathcal{A}^{s}$, pour tout $i, \mathcal{H}^{i}\left(\overline{\mathcal{A}^{s}}\right)$ et $\wedge^{i} \mathcal{H}^{1}(\overline{\mathcal{A}})^{s}$ sont égaux comme sous-faisceaux de $(X \rightarrow \bar{X})_{*} \mathcal{H}^{i}\left(\mathcal{A}^{s}\right)$ munis d'une connexion à pôles logarithmiques.

Démonstration. - En effet, il suffit pour pouvoir appliquer le lemme précédent de vérifier que les $\wedge^{i} \mathcal{H}^{1}(\overline{\mathcal{A}})^{s}$ sont localement libres, il suffit donc de voir que $\mathcal{H}^{1}(\overline{\mathcal{A}})$ est localement libre. Cela se déduit des résultats de [8], qu'on peut appliquer car on a supposé que $\operatorname{dim}_{X} \mathcal{A}<p$. Le cas général se déduit de l'identité précédente.

On notera $\overline{\mathcal{H}}^{i}\left(\mathcal{A}^{s}\right)$ ce faisceau à connexion.

Lemme 3.6. - $\mathcal{H}^{i}\left(\overline{\mathcal{A}_{n}^{s}}\right)$ est localement libre sur $\bar{X}_{n}$, et ne dépend pas de la compactification $\overline{\mathcal{A}^{s}}$.

Démonstration. - En effet, le faisceau $\overline{\mathcal{H}}^{i}\left(\mathcal{A}_{n}^{s}\right)$ est obtenu à partir de $\overline{\mathcal{H}}^{i}\left(\mathcal{A}^{s}\right)$ par changement de base.

On notera dans la suite $\overline{\mathcal{H}}^{i}\left(\mathcal{A}_{n}^{s}\right)$ pour ce faisceau.

\subsubsection{Prolongement de l'action de $E_{s}$}

Il s'agit maintenant de prolonger le morphisme $E_{s} \stackrel{a_{\text {cris }}}{\longrightarrow} \operatorname{End}\left(\mathcal{H}^{\bullet}\left(\mathcal{A}^{s}\right)\right)$ en un morphisme de $\mathbb{Z}_{(p)}$-algèbres $E_{s} \stackrel{a_{\log -\text { cris }}}{\longrightarrow} \operatorname{End}\left(\overline{\mathcal{H}}^{\bullet}\left(\mathcal{A}^{s}\right)\right)$.

La restriction res $: \operatorname{End}\left(\overline{\mathcal{H}}^{\bullet}\left(\mathcal{A}^{s}\right)\right) \rightarrow \operatorname{End}\left(\mathcal{H}^{\bullet}\left(\mathcal{A}^{s}\right)\right)$ est injective. Pour construire $a_{\log -\text { cris }}$, il suffit donc de vérifier que l'image de $a_{\text {cris }}$ est contenue dans l'image de res. Comme $a_{\text {cris }}$ est un morphisme de $\mathbb{Z}_{(p)}$-algèbres, il suffit de vérifier que l'image par $a_{\text {cris }}$ d'une partie génératrice de $E_{s}$ est contenue dans l'image de res. Il s'agit donc de vérifier que l'action des éléments de $\mathcal{G}$ sur les $\mathcal{H}^{\bullet}\left(\mathcal{A}^{s}\right)$ se prolonge en une action sur les $\overline{\mathcal{H}}^{\bullet}\left(\mathcal{A}^{s}\right)$.

Soit $u \in \mathcal{G}$. Supposons d'abord que $u$ soit une matrice ou un élément de $\mathcal{O}_{B}$. Alors $u$ agit sur $\mathcal{A}^{s}$ par une isogénie. D'après la propriété 2, il existe donc deux compactifications $\overline{\mathcal{A}^{s}}{ }_{1}$ et $\overline{\mathcal{A}^{s}}$, et un morphisme $u^{\prime}: \overline{\mathcal{A}}^{s}{ }_{1} \rightarrow \overline{\mathcal{A}}^{s}$ prolongeant l'action de $u$. Alors $u^{\prime}$ fournit l'élément de $\operatorname{End}\left(\overline{\mathcal{H}}^{\bullet}\left(\mathcal{A}^{s}\right)\right)$ voulu. Supposons maintenant qu'on est dans le cas Siegel et que $u$ est de la forme $\theta_{i, j}$. Il suffit de montrer que les morphismes $a_{\text {cris }}\left(\varphi_{i, j}\right)$ et $a_{\text {cris }}\left(\psi_{i, j}\right)$ se prolongent en éléments de $\operatorname{End}\left(\overline{\mathcal{H}}^{\bullet}\left(\mathcal{A}^{s}\right)\right)$. Il existe, d'après l'hypothèse 2 , une compactification $\overline{\mathcal{A}^{s}}$ telle que le faisceau $\left(\mathcal{O}(2)^{\otimes a} \otimes \mathcal{P}_{i, j}\right)^{\otimes b}$ se prolonge en un faisceau $\mathcal{L}$ sur $\overline{\mathcal{A}^{s}}$, avec $a$ et $b$ premiers à $p$. Il existe aussi une compactification ${\overline{\mathcal{A}^{s}}}^{\prime}$ telle que le faisceau $\mathcal{O}(2)^{\otimes c}$ se prolonge en un faisceau $\mathcal{L}^{\prime}$ sur ${\overline{\mathcal{A}^{s}}}^{\prime}$, avec $c$ premier à $p$. Alors l'action de $\left.\frac{1}{b}\left(c_{1}(\mathcal{L})\right)\right)$ est dans $\operatorname{End}\left(\overline{\mathcal{H}}^{\bullet}\left(\mathcal{A}^{s}\right)\right)$, l'action de $-\frac{a}{c}\left(c_{1}\left(\mathcal{L}^{\prime}\right)\right)$ aussi, et l'action 
de $\frac{1}{b}\left(c_{1}(\mathcal{L})\right)-\frac{a}{c}\left(c_{1}\left(\mathcal{L}^{\prime}\right)\right)$ prolonge celle de $a_{\text {cris }}\left(\varphi_{i, j}\right)$. Pour $a_{\text {cris }}\left(\psi_{i, j}\right)$, on fait le même raisonnement, en utilisant la dualité de Poincaré.

On note encore $a_{\log -\text { cris }}$ pour le morphisme $E_{s} \rightarrow \operatorname{End}\left(\overline{\mathcal{H}}^{\bullet}\left(\mathcal{A}_{n}^{s}\right)\right)$ obtenu par réduction.

\subsubsection{Définition de $\overline{\mathcal{F}}$}

Si $V \in \operatorname{Rep}^{a}(G)$, on veut définir $\overline{\mathcal{F}}(V)$ comme le faisceau localement libre muni d'une connexion à pôles logarithmiques intégrable et quasi-nilpotente sur $\bar{X}$ prolongeant $\mathcal{F}(V)$.

$\mathrm{Au}$ vu du paragraphe 3.3.1, il suffit de montrer l'existence de ce prolongement, son unicité et le fait que la construction est fonctorielle étant alors automatiques. Soit $V=q\left(\wedge^{\bullet} \mathcal{V}_{0}^{s}\right)$, où $q$ est un projecteur de $E_{s}$. Il suffit de poser $\overline{\mathcal{F}}(V)=a_{\log -\operatorname{cris}}(q)\left(\overline{\mathcal{H}}^{\bullet}\left(\mathcal{A}^{s}\right)\right)$.

On note $\overline{\mathcal{F}}_{n}(V)$ la réduction modulo $\varpi^{n+1}$ de $\overline{\mathcal{F}}(V)$. Munissons $S_{n}$ de la logstructure triviale, et $\bar{X}_{n}$ de la log-structure provenant du diviseur à croisements normaux $(\bar{X} \backslash X)_{n}$. Alors $\overline{\mathcal{F}}_{n}$ définit un foncteur de $\operatorname{Rep}^{a}(G)$ vers la catégorie des cristaux sur $\left(\bar{X}_{0} / S_{n}\right)_{\text {cris }}^{\log }$, en effet cette catégorie est équivalente à celle des $\mathcal{O}_{\bar{X}_{n}}$-modules munis d'une connexion à pôles logarithmiques intégrable et quasi-nilpotente, $\bar{X}_{n}$ étant un relèvement de $\bar{X}_{0}$ log-lisse sur $S_{n}$.

\section{Structures sur les groupes de cohomologie}

\subsection{Cas étale}

Notons $\bar{K}$ la clôture algébrique de $K$, et $\Gamma=\operatorname{Gal}(\bar{K} / K)$. Le groupe $H_{\mathrm{t}}^{m}\left(X_{\bar{K}}, \mathbb{F}_{n}(V)\right)$ est naturellement muni d'une action de $\Gamma$ car le faisceau $\mathbb{F}_{n}(V)$ est défini sur $X_{K}$.

On aura besoin du lemme suivant pour comparer l'action de Galois sur $H_{\mathrm{t}}^{m}\left(X_{\bar{K}}, \mathbb{F}_{n}(V)\right)$ et sur la cohomologie de $\mathcal{A}_{\bar{K}}^{s}$ :

Lemme 4.1. - Soit $f: Z \rightarrow T$ un morphisme de schémas, $F$ un faisceau constant sur $Z\left(\mathbb{Z} / p^{n} \mathbb{Z}\right.$ ou $\left.\mathbb{Z}_{p}\right)$. Soit $q$ agissant sur $\mathcal{H}^{\bullet}(Z)=R^{\bullet} f_{*} F$ et sur $H^{\bullet}(Z, F)$ de façon compatible avec la suite spectrale de Leray. On suppose que $q$ agit comme un projecteur, dont l'image est entièrement contenue dans $\mathcal{H}^{s}(Z)$. Notons $V=q \mathcal{H}^{\bullet}(Z)$, alors pour tout $m$ on a $H^{m}(T, V)=q H^{m+s}(Z, F)$.

Démonstration. - En effet considérons la suite spectrale de Leray pour calculer la cohomologie de $F$ sur $Z$. On lui applique $q$, on obtient toujours une suite spectrale convergente car $q$ est un projecteur. D'autre part $q H^{m}\left(T, \mathcal{H}^{i}(Z)\right)=$ $H^{m}\left(T, q \mathcal{H}^{i}(Z)\right)$, toujours parce que $q$ est un projecteur. La suite spectrale obtenue a une seule colonne non nulle, dont les termes sont les $H^{m}\left(T, q \mathcal{H}^{s}(Z)\right)$, et aboutit à $q H^{m+s}(Z, F)$, d'où le résultat. 
Notons encore $a_{\mathrm{t}}$ les morphismes $E_{s} \rightarrow \operatorname{End}\left(H_{\mathrm{t}}^{\bullet}\left(\mathcal{A}_{\bar{K}}^{s}, \mathbb{Z}_{p}\right)\right)$ et $E_{s} \rightarrow$ $\operatorname{End}\left(H_{\mathrm{t}}^{\bullet}\left(\mathcal{A}_{\bar{K}}^{s}, \mathbb{Z} / p^{n} \mathbb{Z}\right)\right)$. Supposons que $V=q\left(\wedge^{\bullet} \mathcal{V}_{0}^{s}\right), q$ étant un projecteur homogène de degré $t$, alors on a $H_{\mathrm{t}}^{m}\left(X_{\bar{K}}, \mathbb{F}_{n}(V)\right)=a_{\mathrm{t}}(q) H_{\mathrm{t}}^{m+t}\left(\mathcal{A}_{\bar{K}}^{s}, \mathbb{Z} / p^{n} \mathbb{Z}\right)$. Comme $E_{s}$ agit par des correspondances algébriques définies sur $K$ sur la cohomologie de $\mathcal{A}^{s}$, l'action de $\Gamma$ commute à l'action de $E_{s}$, et la structure galoisienne obtenue sur $H_{\mathrm{t}}^{m}\left(X_{\bar{K}}, \mathbb{F}_{n}(V)\right)$ est compatible à celle sur la cohomologie de $\mathcal{A}_{\bar{K}}$.

\subsection{Cas cristallin}

\subsubsection{Les modules de Fontaine-Laffaille}

On note $\underline{M F_{\text {tor }}^{f}}$ la catégorie suivante. Les objets sont les $\mathcal{O}$-modules $M$ de longueur finie, muni d'une filtration $\mathrm{Fil}^{i} M$ décroissante, telle que $\mathrm{Fil}^{0} M=M$ et $\mathrm{Fil}^{p-1} M=0$, et pour tout $i$, un $\phi_{i}: \mathrm{Fil}^{i} M \rightarrow M \mathcal{O}$-semi-linéaire, vérifiant $\phi_{i \mid \mathrm{Fil}^{i+1} M}=p \phi_{i+1}$, et $\sum_{i} \operatorname{im} \phi_{i}=M$. Les morphismes respectent la filtration et commutent aux $\phi_{i}$.

\subsubsection{La catégorie $M F(\phi)$}

On introduit la catégorie $M F(\phi)$ des $K$-espaces vectoriels munis d'une filtration décroissante et d'un Frobenius. Les objets sont les $K$-espaces vectoriels de dimension finie $M$, muni d'une filtration décroissante $\mathrm{Fil}^{i} M$, et de l'action d'un Frobenius $\phi$ semi-linéaire par rapport au Frobenius $\sigma$ de $K$. Les morphismes doivent commuter au Frobenius, et respecter la filtration.

\subsubsection{Calculs dans un cas particulier}

On se place dans le cas suivant : on a un log-schéma $Z$ qui est propre, et lisse sur $S$ muni de la log-structure triviale.

Soit $n$ un entier positif. On note $S_{n}=\operatorname{Spec} \mathcal{O}_{n}$, muni de la log-structure triviale.

Si $(Z, M)$ est un schéma sur Spec $\mathcal{O}$ muni d'une log-structure $M$, on note $\left(Z_{n}, M_{n}\right)$ le changement de base à $S_{n}$. Si $\mathcal{E}$ est un cristal sur le site $\left.\left(\left(Z_{m}, M_{m}\right) / S_{n}\right)\right)_{\text {cris }}^{\log }$, on notera $H_{\text {cris }}^{i}\left(\left(Z_{m}, M_{m}\right) / S_{n}, \mathcal{E}\right) \quad$ pour $\left.H^{i}\left(\left(\left(Z_{m}, M_{m}\right) / S_{n}\right)\right)_{\text {cris }}^{\log }, \mathcal{E}\right)$, et $H_{\text {cris }}^{i}\left(\left(Z_{m}, M_{m}\right) / S_{n}\right)$ pour $H_{\text {cris }}^{i}\left(\left(Z_{m}, M_{m}\right) / S_{n}\right.$, $\left.\mathcal{O}_{Z_{m} / S_{n}}\right)$. On omettra la mention de la log-structure si cela ne cause pas de confusion. Remarquons que pour tout $m \leq n$, les $H_{\text {cris }}^{i}\left(\left(Z_{m}, M_{m}\right) / S_{n}\right)$ ne dépendent pas de $m$, on notera $H_{\text {cris }}^{i}\left((Z, M) / S_{n}\right)$ leur valeur commune. Enfin on note $H_{\text {cris }}^{i}((Z, M) / S)=\lim _{\leftarrow} H_{\text {cris }}^{i}\left((Z, M) / S_{n}\right)$.

On a les deux résultats suivants :

Proposition 4.2. - Pour tout $0 \leq i \leq p-2, H_{\text {cris }}^{i}\left((Z, M) / S_{n}\right)$ est un module de Fontaine-Laffaille. Pour tout $i \geq 0, H_{\text {cris }}^{i}((Z, M) / S) \otimes K$ est un élément de $M F(\phi)$. 
Démonstration. - La preuve de la première partie de la proposition est identique à celle de l'article [5], qui traite le cas où $Z$ est muni de la log-structure triviale.

Notons $S_{n}^{\prime}$ le log-schéma dont le schéma sous-jacent est le même que $S_{n}$, et dont la log-structure provient de $\mathbb{N} \rightarrow \mathcal{O}_{n}, 1 \mapsto 0$. Il s'agit de la même log-structure que celle définie dans [7], paragraphe 3.4. Notons $\left(Z^{\prime}, M^{\prime}\right)$ le log-schéma déduit de $(Z, M)$ par le changement de base $S_{n}^{\prime} \rightarrow S_{n}$. Alors $H_{\text {cris }}^{i}\left((Z, M) / S_{n}\right)$ et $H_{\text {cris }}^{i}\left(\left(Z^{\prime}, M^{\prime}\right) / S_{n}^{\prime}\right)$ sont canoniquement isomorphes pour tout $i$. Les $H_{\text {cris }}^{i}\left(\left(Z^{\prime}, M^{\prime}\right) / S_{n}^{\prime}\right)$ sont munis d'un Frobenius et d'un opérateur de monodromie, définis dans [7], paragraphe 3. L'opérateur de monodromie est ici nul, $\left(Z^{\prime}, M^{\prime}\right)$ provenant par changement de base de $(Z, M)$ qui est log-lisse sur $S_{n} . H_{\text {cris }}^{i}\left(\left(Z^{\prime}, M^{\prime}\right) / S^{\prime}\right) \otimes K$, et donc aussi $H_{\text {cris }}^{i}((Z, M) / S) \otimes K$ est ainsi naturellement muni d'une structure d'élément de $M F(\phi)$.

\subsubsection{Action des endomorphismes sur le prolongement des cristaux}

Notons $\bar{H}^{i}\left(\mathcal{A}^{s} / S_{n}\right)$ la limite des $H_{\text {cris }}^{i}\left(\overline{\mathcal{A}}_{n} / S_{n}\right)$, pour les $\overline{\mathcal{A}^{s}}$ de notre famille de compactifications. Alors :

Proposition 4.3. - Le morphisme $H_{\text {cris }}^{i}\left(\overline{\mathcal{A}}_{n}{ }_{n} / S_{n}\right) \rightarrow \bar{H}^{i}\left(\mathcal{A}^{s} / S_{n}\right)$ est un isomorphisme pour toute compactification $\overline{\mathcal{A}^{s}}$ et pour tout $n$.

Démonstration. - Il suffit pour cela de voir que tout morphisme entre compactifications qui est l'identité sur $\mathcal{A}^{s}$ induit un isomorphisme entre les groupes de cohomologie.

Considérons la suite spectrale de Leray :

$$
E_{2}^{i, j}=H^{i}\left(\bar{X}_{n} / S_{n}, R^{j} f_{s \mathrm{cris} *} \mathcal{O}_{\overline{\mathcal{A}}_{n} / S_{n}}\right) \Rightarrow H^{i+j}\left(\overline{\mathcal{A}}_{n} / S_{n}\right)
$$

Un morphisme entre deux compactifications induit un morphisme de suites spectrales, qui est un isomorphisme sur les $E_{2}^{i, j}$, donc aussi sur l'aboutissement.

On cherche à définir un morphisme de $\mathbb{Z}_{(p)}$-algèbres $E_{s} \rightarrow \operatorname{End}\left(\bar{H}^{i}\left(\mathcal{A}^{s} / S_{n}\right)\right)$.

On a la suite spectrale suivante, qu'on appellera encore suite spectrale de Leray, qui provient de n'importe quelle compactification $\overline{\mathcal{A}^{s}}$ de $\mathcal{A}^{s}$ :

$$
E_{2}^{i, j}=H^{i}\left(\bar{X}_{n} / S_{n}, \overline{\mathcal{H}}^{j}\left(\mathcal{A}_{n}^{s}\right)\right) \Rightarrow \bar{H}^{i+j}\left(\mathcal{A}^{s} / S_{n}\right)
$$

Proposition 4.4. - Il existe un unique morphisme $a_{\log \text {-cris }}$ de $\mathbb{Z}_{(p)}$-algèbres $E_{s} \rightarrow \operatorname{End}\left(\bar{H}^{i}\left(\mathcal{A}^{s} / S_{n}\right)\right)$ tel que l'action de $E_{s}$ sur les $\overline{\mathcal{H}}^{j}\left(\mathcal{A}_{n}^{s}\right)$ et sur les $\bar{H}^{i}\left(\mathcal{A}^{s} / S_{n}\right)$ donnée par $a_{\log -\mathrm{cris}}$ soit compatible à la suite spectrale de Leray. 
Démonstration. - On commence par définir l'image de l'ensemble $\mathcal{G}$ des éléments géométriques de $E_{s}$, et on montre ensuite que l'on peut prolonger en un morphisme de $\mathbb{Z}_{(p)}$-algèbres.

Pour définir l'image d'un élément de $\mathcal{G}$, on fait exactement comme dans le paragraphe 3.3.2. Il faut voir que le choix fait est unique. Cela provient du fait que l'action de $u \in \mathcal{G}$ sur les termes $E_{2}^{i, j}$ de la suite spectrale ne dépend pas des choix faits, comme expliqué en 3.3.2, et de la compatibilité de l'action du prolongement à la suite spectrale de Leray.

Il reste à voir que l'action des éléments de $\mathcal{G}$ se prolonge en un morphisme d'algèbres $E_{s} \rightarrow \operatorname{End}\left(\bar{H}^{i}\left(\mathcal{A}^{s} / S_{n}\right)\right)$. Cela provient encore une fois de la compatibilité avec la suite spectrale de Leray, et du fait que $E_{s} \rightarrow \operatorname{End}\left(\overline{\mathcal{H}}^{\bullet}\left(\mathcal{A}^{s} / S_{n}\right)\right)$ est un morphisme d'algèbres.

\subsubsection{La cohomologie des cristaux}

Posons $H_{\text {cris }}^{i}(\bar{X} / S, \overline{\mathcal{F}}(V))={\underset{\leftarrow}{n}}_{\lim _{\text {cris }}} H_{n}^{i}\left(\bar{X}_{n} / S_{n}, \overline{\mathcal{F}}_{n}(V)\right)$. On a le résultat suivant :

Proposition 4.5. - Pour tout $V \in \operatorname{Rep}^{a}(G), \quad$ pour tout $i$, $H_{\text {cris }}^{i}(\bar{X} / S, \overline{\mathcal{F}}(V)) \otimes K$ est un élément de $M F(\phi)$. Pour tout $V \in \operatorname{Rep}_{A}^{a}(G)$ homogène de degré $t$, pour tout $i$ tel que $i+t \leq p-2$, pour tout $n$, $H_{\text {cris }}^{i}\left(\bar{X}_{n} / S_{n}, \overline{\mathcal{F}}_{n}(V)\right)$ est un élément de $\underline{M F}_{\text {tor }}^{f}$.

Démonstration. - En effet, soit $V=q\left(\wedge^{\bullet} \mathcal{V}_{0}^{s}\right)$, avec $q$ homogène de degré $t$, on a alors de façon similaire au lemme 4.1 les égalités $H_{\text {cris }}^{i}(\bar{X} / S, \overline{\mathcal{F}}(V)) \otimes K=$ $a_{\text {cris }}(q) \bar{H}_{\log -\text { cris }}^{i+t}\left(\mathcal{A}^{s} / S\right) \otimes K$ et $H_{\text {cris }}^{i}\left(\bar{X}_{n} / S_{n}, \overline{\mathcal{F}}_{n}(V)\right)=a_{\text {cris }}(q) \bar{H}_{\text {cris }}^{i+t}\left(\mathcal{A}_{n}^{s} / S_{n}\right)$. Il reste à voir que l'action de $E_{s}$ par $a_{\mathrm{log}-\text { cris }}$ respecte les structures d'élément de $M F(\phi)$, et que l'action de $E(A)_{s}$ respecte les structures de module de FontaineLaffaille, ce qui se voit sur les éléments géométriques.

\section{Théorème de comparaison}

\subsection{Le cas des faisceaux constants}

Notons $\operatorname{Rep}_{\mathbb{Z}_{p}}(\Gamma)$ la catégorie des $\mathbb{Z}_{p}$-représentations de type fini de $\Gamma=$ $\operatorname{Gal}(\bar{K} / K)$. Nous avons un foncteur contravariant et pleinement fidèle : $V_{\text {cris }}$ : $M F_{\text {tor }}^{f} \rightarrow \operatorname{Rep}_{\mathbb{Z}_{p}}(\Gamma)$ qui est défini par $V_{\text {cris }}(M)=\operatorname{Hom}\left(M, A_{\text {cris }, \infty}\right)$. L'anneau $A_{\text {cris }}$ est défini dans [1], 6.3, et $A_{\text {cris }, \infty}=A_{\text {cris }} \otimes \mathbb{Q}_{p} / \mathbb{Z}_{p}$. L'anneau $A_{\text {cris }}$ est muni d'une filtration décroissante et d'un Frobenius, et les homomorphismes que l'on considère doivent être compatibles à la filtration et à l'action du Frobenius.

Soit $Z$ un schéma propre et lisse sur $\operatorname{Spec} \mathcal{O}$, et $D$ un diviseur à croisements normaux relatifs de $Z, U$ l'ouvert complémentaire de $D$. On munit $Z$ de la $\log$-structure $M$ définie par le diviseur $D$, et $\operatorname{Spec} \mathcal{O}$ de la log-structure triviale.

TOME $137-2009-\mathrm{N}^{\mathrm{O}} 3$ 
Proposition 5.1. - Pour $0 \leq m \leq p-2$, on a un isomorphisme canonique compatible à l'action de Galois :

$$
V_{\text {cris }}\left(H_{\text {cris }}^{m}\left((Z, M) / S_{n}\right)\right)=H_{\mathrm{t}}^{m}\left(U_{\bar{K}}, \mathbb{Z} / p^{n} \mathbb{Z}\right)^{\vee}
$$

Proposition 5.2. - Pour tout $m$, il existe un isomorphisme canonique qui respecte l'action de $\Gamma$, la filtration et le Frobenius :

$$
\gamma_{m}: B_{\text {cris }} \otimes_{\mathcal{O}} H_{\text {cris }}^{m}((Z, M) / S) \stackrel{\sim}{\longrightarrow} B_{\text {cris }} \otimes_{\mathbb{Q}_{p}} H_{\mathrm{t}}^{m}\left(U_{\bar{K}}, \mathbb{Q}_{p}\right)
$$

Démonstration. - Le résultat de la proposition 5.1 provient de travaux de Breuil ([2]) et Tsuji ([16]). Ces résultats s'appliquent dans un cadre beaucoup plus général que celui considéré ici, et décrivent une comparaison entre la cohomologie étale de $U_{\bar{K}}$ et la cohomologie de $\left(Z^{\prime}, M^{\prime}\right) / E_{n}$. Ici $\left(Z^{\prime}, M^{\prime}\right)$ est obtenu comme dans le paragraphe 4.2.3 par changement de base de $(Z, M)$ de $S_{n}$ à $S_{n}^{\prime}$. $E_{n}$ est le log-schéma dont le schéma sous-jacent est $\operatorname{Spec} \mathcal{O}_{n}\langle u\rangle$, l'enveloppe à puissances divisées de l'algèbre $\mathcal{O}_{n}[u]$ des polynômes en l'indéterminée $u$, muni de la log-structure associée à $\mathbb{N} \rightarrow \mathcal{O}_{n}\langle u\rangle, 1 \mapsto u$. Dans notre cas particulier, on a une relation simple entre la cohomologie de $\left(Z^{\prime}, M^{\prime}\right) / E_{n}$ et celle de $\left(Z^{\prime}, M^{\prime}\right) / S_{n}^{\prime}$, donnée par $H_{\text {cris }}^{m}\left(\left(Z^{\prime}, M^{\prime}\right) / E_{n}\right)=\mathcal{O}_{n}\langle u\rangle \otimes H_{\text {cris }}^{m}\left(\left(Z^{\prime}, M^{\prime}\right) / S_{n}^{\prime}\right)$, qui nous permet d'obtenir le résultat de la proposition 5.1.

Pour la version rationnelle 5.2, le résultat provient de résultats de Tsuji ([15], voir aussi [17]). Comme dans le cas de torsion, la situation se simplifie par rapport au cas général, du fait qu'ici la monodromie agissant sur $H_{\text {cris }}^{m}((Z, M) / S) \otimes K$ est nulle.

\subsection{Les théorèmes}

Énonçons maintenant nos théorèmes. Le premier concerne la cohomologie

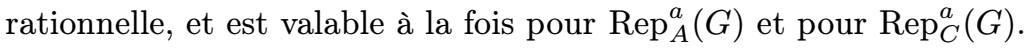

ThÉORÈme 5.3. - Soit $V \in \operatorname{Rep}^{a}(G)$, il existe un isomorphisme

$$
\gamma: B_{\text {cris }} \otimes_{\mathcal{O}} H_{\log -\text { cris }}^{m}(\bar{X} / S, \overline{\mathcal{F}}(V)) \stackrel{\sim}{\longrightarrow} B_{\text {cris }} \otimes_{\mathbb{Z}_{p}} H_{\mathrm{t}}^{m}\left(X_{\bar{K}}, \mathbb{F}(V)\right)
$$

Le second concerne la torsion de la cohomologie. Il n'est valable que dans le cas de la catégorie $\operatorname{Rep}_{A}^{a}(G)$, et non dans la catégorie $\operatorname{Rep}_{C}^{a}(G)$. La raison de cette limitation vient du lemme 5.5 : contrairement au cas rationnel, nous ne savons pas si l'isomorphisme de comparaison à coefficients constants est compatible avec la dualité de Poincaré et les structures produits. Si nous disposions d'un tel résultat le théorème serait valable aussi dans le cas de la catégorie $\operatorname{Rep}_{C}^{a}(G)$. 
ThÉORÈme 5.4. - Soit $V \in \operatorname{Rep}_{A}^{a}(G)$, et $m$ tel que $m+t(V) \leq p-2$. $H_{\mathrm{t}}^{m}\left(X_{\bar{K}}, \mathbb{F}_{n}(V)\right)$ est muni d'une action du groupe de Galois $\Gamma, H_{\mathrm{cris}}^{m}\left(\bar{X}_{n} / S_{n}\right.$, $\left.\overline{\mathcal{F}}_{n}(V)\right)$ est muni d'une structure de module de Fontaine-Laffaille, et on a un isomorphisme :

$$
V_{\text {cris }}\left(H_{\text {cris }}^{m}\left(\bar{X}_{n} / S_{n}, \overline{\mathcal{F}}_{n}(V)\right)\right)=H_{\mathrm{t}}^{m}\left(X_{\bar{K}}, \mathbb{F}_{n}(V)\right)^{\vee}
$$

Notons qu'on peut déduire de ce théorème comme dans l'article [2], para-

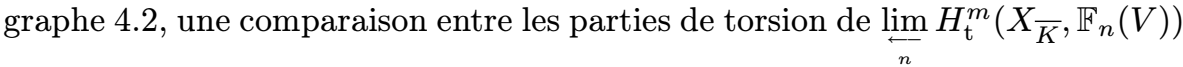
et $\operatorname{le}_{\underset{n}{\lim }} H_{\text {cris }}^{m}\left(\bar{X}_{n} / S_{n}, \overline{\mathcal{F}}_{n}(V)\right)$, ainsi qu'une comparaison entre leurs parties libres.

Le point essentiel de la preuve dans les deux cas est le résultat suivant :

Lemme 5.5. - Soit $u \in E(A)_{s} . u$ agit $\operatorname{sur} \bar{H}^{m}\left(\mathcal{A}^{s} / S_{n}\right)$ et $\operatorname{sur} H_{\mathrm{t}}^{m}\left(\mathcal{A}_{\bar{K}}^{s}, \mathbb{Z} / p^{n} \mathbb{Z}\right)$ $(m \leq p-2)$ de façon compatible avec l'isomorphisme $V_{\text {cris. }}$. Soit $u \in E_{s}, u$ agit sur $\bar{H}^{m}\left(\mathcal{A}^{s} / S\right) \otimes \mathbb{Q}$ et sur $H_{\mathrm{t}}^{m}\left(\mathcal{A}_{\bar{K}}^{s}, \mathbb{Q}_{p}\right)$ de façon compatible avec l'isomorphisme $\gamma_{m}$ du théorème 5.2.

Démonstration. - Il suffit de montrer la compatibilité des actions pour l'ensemble des éléments géométriques $\mathcal{G}$ de $E_{s}$, puisqu'ils engendrent $E_{s}$ comme $\mathbb{Z}_{(p) \text {-algèbre. }}$

Soit $u$ un élément de $E_{s}$ provenant d'une matrice de déterminant non nul, ou d'un élément non nul de $\mathcal{O}_{B}$. Son action sur la cohomologie provient d'une isogénie de $\mathcal{A}^{s}$, qu'on notera encore $u$. D'après la propriété $2 u$ se prolonge en un morphisme entre deux compactifications $u: \overline{\mathcal{A}}_{1} \rightarrow \overline{\mathcal{A}}^{s}$. D'où par fonctorialité de $V_{\text {cris }}, V_{\text {cris }}\left(u: H_{\text {cris }}^{m}\left(\left(\overline{\mathcal{A}}^{\vee}\right)_{n} / S_{n}\right) \rightarrow H_{\text {cris }}^{m}\left(\left(\overline{\mathcal{A}}_{1}\right)_{n} / S_{n}\right)=\left(u^{\vee}\right.\right.$ : $\left.H_{\mathrm{t}}^{m}\left(\mathcal{A}_{\bar{K}}^{s}, \mathbb{Z} / p^{n} \mathbb{Z}\right)^{\vee} \rightarrow H_{\mathrm{t}}^{m}\left(\mathcal{A}_{\bar{K}}^{s}, \mathbb{Z} / p^{n} \mathbb{Z}\right)^{\vee}\right)$, ce qui est bien la compatibilité voulue. De même, on a aussi la compatibilité pour l'action sur $H_{\mathrm{t}}^{m}\left(U_{\bar{K}}, \mathbb{Q}_{p}\right)$ et $H_{\text {cris }}^{m}((X, M) / S) \otimes K$.

Dans le cas Siegel, il faut aussi considérer les éléments de la forme $\theta_{i, j}$. Il s'agit donc de voir que l'action des $\varphi_{i, j}$ et des $\psi_{i, j}$ sur $H_{\mathrm{t}}^{m}\left(U_{\bar{K}}, \mathbb{Q}_{p}\right)$ et $H_{\text {cris }}^{m}((X, M) / S) \otimes K$ est compatible. Cela provient du fait que l'isomorphisme de comparaison 5.2 fait correspondre les classes de Chern ([15]) et est compatible aux structures produit sur les groupes de cohomologie et à la dualité de Poincaré ([17]).

Démonstration des théorèmes 5.3 et 5.4. - Montrons le théorème 5.4. Soit $V \in \operatorname{Rep}^{a}(G)$, on peut supposer qu'il existe un entier $s$, et un projecteur $q$ dans $E(A)_{s}$ de degré $t$, tels que $V=q\left(\wedge^{\bullet} \mathcal{V}_{0}^{s}\right)$. Le théorème de comparaison s'applique car $m+t \leq p-2$, et nous donne un isomorphisme $V_{\text {cris }}\left(\bar{H}^{m+t}\left(\mathcal{A}^{s} / S_{n}\right)\right)=H_{\mathrm{t}}^{m+t}\left(\mathcal{A}_{\bar{K}}^{s}, \mathbb{Z} / p^{n} \mathbb{Z}\right)^{\vee}$.

TOME $137-2009-\mathrm{N}^{\circ} 3$ 
Appliquons $q$ : comme l'action de $E(A)_{s}$ commute à $V_{\text {cris }}$ on a donc un isomorphisme : $V_{\text {cris }}\left(a_{\log -\operatorname{cris}}(q) \bar{H}^{m+t}\left(\mathcal{A}^{s} / S_{n}\right)\right)=\left(a_{\mathrm{t}}(q) H_{\mathrm{t}}^{m+t}\left(\mathcal{A}_{\bar{K}}^{s}, \mathbb{Z} / p^{n} \mathbb{Z}\right)\right)^{\vee}$.

D'après le lemme 4.1 , cela donne : $V_{\text {cris }}\left(H_{\log -\text { cris }}^{m}\left(\bar{X}, \overline{\mathcal{F}}_{n}(V)\right)\right)=H_{\mathrm{t}}^{m}\left(X_{\bar{K}}\right.$, $\left.\mathbb{F}_{n}(V)\right)^{\vee}$.

La preuve du théorème 5.3 est identique.

\section{BIBLIOGRAPHIE}

[1] C. Breuil - « Topologie log-syntomique, cohomologie log-cristalline et cohomologie de Čech », Bull. Soc. Math. France 124 (1996), p. 587-647.

[2] _ _ Cohomologie étale de $p$-torsion et cohomologie cristalline en réduction semi-stable », Duke Math. J. 95 (1998), p. 523-620.

[3] P. Deligne - Équations différentielles à points singuliers réguliers, Lecture Notes in Math., vol. 163, Springer, 1970.

[4] G. Faltings \& C.-L. Chai - Degeneration of abelian varieties, Ergebnisse Math. Grenzg. (3), vol. 22, Springer, 1990.

[5] J.-M. Fontaine \& W. Messing - «p-adic periods and $p$-adic étale cohomology », in Current trends in arithmetical algebraic geometry (Arcata, Calif., 1985), Contemp. Math., vol. 67, Amer. Math. Soc., 1987, p. 179207.

[6] W. Fulton \& J. HARRIS - Representation theory, Graduate Texts in Math., vol. 129, Springer, 1991.

[7] O. Hyodo \& K. Kato - « Semi-stable reduction and crystalline cohomology with logarithmic poles », Astérisque 223 (1994), p. 221-268.

[8] L. ILlusie - «Réduction semi-stable et décomposition de complexes de de Rham à coefficients », Duke Math. J. 60 (1990), p. 139-185.

[9] J. C. JANTZEN - Representations of algebraic groups, Pure and Applied Mathematics, vol. 131, Academic Press Inc., 1987.

[10] R. E. Kоттwitz - «Points on some Shimura varieties over finite fields », J. Amer. Math. Soc. 5 (1992), p. 373-444.

[11] K.-W. LAN - «Arithmetic compactifications of PEL-type shimura varieties », Thèse, Harvard University, 2008.

[12] A. Mokrane \& J. Tilouine - «Cohomology of Siegel varieties with p-adic integral coefficients and applications », Astérisque 280 (2002), p. 195.

[13] P. Polo \& J. Tilouine - «Bernstein-Gelfand-Gelfand complexes and cohomology of nilpotent groups over $\mathbb{Z}_{(p)}$ for representations with $p$-small weights », Astérisque 280 (2002), p. 97-135. 
[14] S. Rozensztajn - «Compactification de schémas abéliens dégénérant au-dessus d'un diviseur régulier », Doc. Math. 11 (2006), p. 57-71.

[15] T. TsuJI - «p-adic étale cohomology and crystalline cohomology in the semi-stable reduction case », Invent. Math. 137 (1999), p. 233-411.

[16] _ « On $p$-adic nearby cycles of log smooth families », Bull. Soc. Math. France 128 (2000), p. 529-575.

[17] G. YAmAshita - «p-adic étale cohomology and crystalline cohomology for open varieties », Sūrikaisekikenkyūsho Kōkyūroku 1324 (2003), p. 130-141. 